\title{
Determinantes psicológicos e situacionais do comportamento de conservação de água: um modelo estrutural ${ }^{1}$
}

\author{
Víctor Corral-Verdugo \\ Universidad de Sonora, México
}

\begin{abstract}
Resumo
Um modelo de fatores psicológicos e situacionais que afetam o consumo de água foi especificado e testado. Foram sujeitos deste estudo 500 indivíduos moradores de duas cidades do estado de Sonora, no México. Uma dessas cidades sofre escassez crônica de água, enquanto a outra tem provisão suficiente do líquido. Foram realizadas observações diretas do consumo de água e de habilidades para conservação de indivíduos em diferentes atividades. Através de um questionário também foram avaliadas crenças utilitárias relativas ao uso de água e motivos para sua conservação. A posse de utensílios que envolvem o uso de água foi considerada como um fator situacional que promove seu consumo, ao passo que a escassez de água foi considerada um inibidor potencial desse consumo. Estas variáveis foram incorporadas em um modelo estrutural, cujos resultados revelaram que o consumo de água foi significativa e positivamente influenciado por crenças utilitárias e pela posse de equipamentos domésticos facilitadores do consumo, enquanto que motivos e habilidades de conservação bem como escassez de água inibiam tal consumo. Motivos de conservação foram positivamente afetados pela escassez de água e habilidades de conservação, e negativamente influenciados por crenças utilitárias. Covariâncias significativas entre os determinantes de consumo de água revelaram a interdependência de variáveis situacionais e psicológicas ao influenciarem o uso da água.
\end{abstract}

Palavras-chave: conservação de água; psicologia ambiental; variáveis situacionais; modelos de equações estruturais.

\begin{abstract}
Psychological and situational determinants of water conservation behavior: a structural model. A model of psychological and situational factors affecting water consumption was specified and tested. 500 individuals living at two cities of Sonora, Mexico were the subjects for this study. One of those cities experienced a chronic scarcity of water, while the other one had a sufficient supply of the liquid. Direct observations of individuals' water consumption (in different activities) and of their conservation skills were conducted. Also, their utilitarian beliefs regarding the use of water, and their conservation motives were assessed using a questionnaire. The possession of furniture for using water was considered as one situational factor promoting water consumption, and water scarcity was considered as a potential inhibitor of that consumption. These variables were incorporated in a structural model. Results of this model revealed that water consumption was significantly and positively influenced by utilitarian beliefs and the possession of furniture, while conservation motives, conservation skills and water scarcity inhibited such consumption. Conservation motives were positively affected by water scarcity and conservation skills, and negatively influenced by utilitarian beliefs. Significant covariances between the determinants of water consumption revealed the interdependence of situational and psychological variables affecting water use.
\end{abstract}

Key words: water conservation; environmental psychology; situational variables; structural equation models.

A água é talvez o recurso natural mais valioso para a humanidade. Sua escassez representa atualmente um problema ambiental crescente para a população (Gardner, 2002; Prince of Orange \& Riijsberman, 2000). Realmente, a escassez de água pode se apresentar como a ameaça ambiental mais séria para humanidade no século 21 (Brown \& Flavin, 1999). Somerville e Briscoe (2001) declaram: "sistemas de água estão sob severa tensão em muitas partes do mundo com espelhos d’água em partes de México, Índia, China e norte da África que recuam até $1 \mathrm{~m}$ por ano” (p. 2217). De acordo com Middlestadt et al. (2001), conservação e escassez de água ameaçam causar uma crise nacional na Jordânia, neste novo século.

Uma grande quantidade de pessoas no mundo sofre de escassez de água: milhões de crianças estão morrendo todos os anos de diarréia e a metade dos pantanais do mundo foi 
destruída durante o último século. Também a metade dos rios do mundo é poluída, muitos estão agonizantes e não chegam ao mar (Crosgrove \& Rijsberman, 2000). No México, a Comissão Nacional de Água declarou recentemente que, a menos que uma temporada extraordinária de chuva acontecesse, este país teria água para poucos meses. Embora no México a maior parte da água seja empregada na agricultura, o crescimento acelerado das cidades resultou num significativo aumento do consumo doméstico de água. Esta tendência está acontecendo na maioria das nações em desenvolvimento, forçando os governos a implementarem estratégias de conservação de água em áreas urbanas. Soluções para este desafio têm de ser encontradas combinando estratégias tecnológicas e sóciocomportamentais para promover a conservação da água em populações de todo o mundo.

O comportamento humano é considerado a principal causa de deterioração ambiental, por isso precisamos entender as causas do comportamento ambientalmente relevante (Oskamp, 2000). De acordo com Fishbein, Middlestadt e Hitchcock (1991), "a chave do sucesso para as intervenções comportamentais é a identificação dos determinantes específicos daqueles comportamentos que se quer manter ou mudar” (p. 243). Assim, estratégias efetivas que promovam a conservação de água deveriam envolver esses determinantes (Middlestadt et al., 2001).

O problema da escassez de água possui componentes psicológicos e sociais. As pessoas desperdiçam água influenciadas por motivos, crenças, percepções e normas pessoais (Corral-Verdugo, 2002). Fatores situacionais, tais como disponibilidade de utensílios ou equipamento para consumo de água, tamanho das famílias e disponibilidade de recursos financeiros, também promovem o desperdício de água (Aitken, McMahon, Wearing \& Finlayson, 1994). Portanto, o estudo de determinantes psicológicos e situacionais do comportamento de conservação de água é necessário para se entender quais características pessoais e quais fatores situacionais poderiam ser úteis para a promoção de padrões de consumo responsável de água.

\section{Determinantes psicológicos da conservação de água}

Não há muita informação sobre preditores psicológicos de conservação de água. Atividades como reciclagem (Daneshvary, Daneshvary \& Schwer, 1998; Ewing, 2001) e conservação de energia (Gatersleben, Steg \& Vlek, 2002) estão entre os tipos mais investigados de comportamento próambiental, em detrimento do estudo de outras ações, como conservação de água. Não obstante, existem vários estudos nessa área, o que permite a construção de um quadro de referência para o estudo de preditores disposicionais de economia de água.

Motivos para economia de água estão entre o preditores significativos de ações pró-ambientais. Algumas pessoas se engajam em conservação de água para economizar esse recurso, por cooperar com alguma campanha de conservação (Corral-Verdugo, 2002), ou por pagar menos pelo recurso consumido (López, Balboa, Igartúa \& Claramunt, 1994). A puni- ção por consumo excessivo também induz à conservação de água (Agras, Jacob \& Ledebeck, 1980). Em geral, a literatura mostra que quanto mais motivos uma pessoa tem para economizar água, mais ela conserva esse recurso.

Habilidades também são determinantes importantes de conservação da água. Corral-Verdugo (2002) constatou que as habilidades da população para economizar água eram indicadores de competência de conservação, um fator que influencia diretamente o comportamento de conservação de água, enquanto Middlestadt et al. (2001) indicaram que proporcionar a estudantes do ensino médio "conhecimento sobre comportamentos” específicos de como economizar água, pode levar a comportamentos de conservação do líquido. Estes resultados indicam que oferecer às pessoas habilidades específicas sobre como economizar o líquido, é um passo importante e necessário para conduzir seu comportamento em direção à uma meta de conservação.

\section{Determinantes situacionais}

Situações físicas, normativas ou facilitadoras também promovem o desperdício ou a conservação de água. Na Austrália, Aitken et al. (1994) estabeleceram que o consumo de água dependia principalmente da condição sócio-econômica da família e de seu tamanho. Famílias maiores, morando em casas caras, consomem mais água em comparação aos casos opostos. Hanke e Mare (1982), na Suécia, também chegaram a resultados semelhantes. Além disso, Syme, Thomas e Salerian (1983) relataram que o valor do jardim, a renda econômica e a percepção dos usuários em relação ao valor dos seus jardins eram preditores positivos de consumo de água. Nos EUA, De Oliver (1999) constatou que uma campanha de conservação voluntária de água não afetou o consumo das pessoas de classes sócio-econômicas mais altas. Entretanto, quando essa campanha se tornou obrigatória, aquelas pessoas cooperaram com o esforço de conservação exigido.

Também nos EUA, Geller, Erickson e Buttram (1983) consideraram que dispositivos econômicos para o uso de água eram significativamente efetivos na redução do seu consumo. No México, Corral-Verdugo (2002) investigou o efeito da carência de disponibilidade de água. Os seus resultados revelaram que a escassez de água é um dos fatores situacionais mais importantes a influir no esforço de conservação de água. Em resumo, esses resultados indicam que situações como ter dinheiro, casas e jardins grandes e ainda uma família grande induzem ao desperdício de água; enquanto o uso de dispositivos de economia de água, enfrentar normas que exigem um consumo reduzido e morar em lugares onde a água já é naturalmente escassa induzem à conservação.

A maioria dos estudos revisados acima investigou aspectos psicológicos ou situacionais que conduzem ao consumo ou à conservação de água. Estudar o efeito combinado de ambos os tipos de fatores poderia ser necessário para se obter uma visão mais completa de como os indivíduos são compelidos a economizar o líquido. Também é importante saber como os preditores de conservação de água se relacionam entre si. Por conseguinte, este artigo apresenta um estudo em que foram investigados padrões de consumo de água 
e analisado o efeito combinado de variáveis situacionais e disposicionais sobre aquele consumo.

\section{Método}

\section{Participantes e locais}

Participaram deste estudo 500 cidadãos residentes em Hermosillo $(\mathrm{N}=389)$ e Ciudad Obregon $(\mathrm{N}=111)$, duas cidades do estado de Sonora, no noroeste do México. Para a amostragem dos residentes, foram consideradas três faixas representativas das classes sócio-econômicas baixa, média e alta, com base na classificação do Instituto Nacional de Geografia e Informática (Instituto Nacional de Geografía e Informática, 1992). Foram selecionados aleatoriamente 130 domicílios de Hermosillo e 40 de Ciudad Obregón, estratificados em classe mais alta (15\%), classe média (40\%) e classe mais baixa (45\%). Esses estratos representam a composição sócio-econômica de ambas as comunidades. De cada grupo familiar eram selecionados três integrantes: a dona de casa, seu marido (ou qualquer outro adulto, caso ela não tivesse marido) e uma seleção aleatória de jovens na faixa de 10 a 17 anos.

No total havia 201 pessoas do sexo masculino e 299 do feminino, com uma média de 35,9 anos de idade e com um número médio de 10,4 anos de escolaridade. O número médio de moradores por domicílio foi de 4,7 pessoas e a média geral de renda mensal foi de 680 dólares. A distribuição de renda mostrou-se assimétrica, positivamente desviada e com ampla dispersão, indicando que a maioria das famílias tem renda baixa, uma característica das cidades mexicanas (Instituto Nacional de Geografía e Informática, 1992). A Tabela 1 mostra essas variáveis demográficas para ambas as amostras, fazendo ver que não há diferenças significativas entre elas.

\section{Instrumentos}

Foi administrado um instrumento que investigava crenças utilitárias de água, motivos para sua conservação e variáveis demográficas. Também foram realizados registros e observações diretas do uso de água em atividades variadas, assim como observação da quantidade de aparelhos e uten- sílios domésticos que consomem água (caldeiras, chuveiros, privadas, torneiras, coolers e caixas d’água) presentes em todas as residências.

Habilidades de conservação de água também foram registradas sob a forma do número de ações efetivas que resultam na sua economia. Solicitou-se aos participantes envolvidos em atividades de consumo de água (lavar carro, regar plantas) ou que relatavam como agiam em outras (como tomar um banho de chuveiro), que economizassem tanta água quanto possível. As crenças utilitárias foram analisadas com base no grau de concordância com afirmações em que a água é considerada como um recurso inesgotável e barato, assegurando que os cientistas podem resolver o problema de sua escassez. Esses itens permitiam respostas numa escala de 4 (concordância total) a 1 (discordância total). Motivos para conservação de água, por sua vez, foram investigados a partir das razões que as pessoas davam para conservar o líquido. Várias razões foram listadas em uma folha de papel e os participantes manifestavam sua concordância com elas, utilizando uma escala de 0 (discordância total) a 10 (concordância total).

\section{Procedimento}

Os pesquisadores abordavam as donas de casa para obter sua concordância em participar. Pediam que elas observassem quanto tempo elas próprias e os outros moradores do domicílio gastavam no consumo de água em várias atividades. Após uma breve sessão de treinamento, as donas de casa faziam três observações do consumo de água durante a semana seguinte. Ao término da última observação, cada participante respondeu às escalas e forneceu informações demográficas.

\section{Análise de dados}

Foram obtidas estatísticas univariadas para cada variável investigada, assim como os “Alfas de Cronbach” das escalas psicológicas e comportamentais (crenças, motivos, habilidades, consumo de água). Foram efetuados testes $t$ para avaliar diferenças de médias entre as cidades, em relação às variáveis psicológicas/comportamentais e situacionais. E, fi-

Tabela 1

Características demográficas das amostras

\begin{tabular}{llllc}
\hline \multicolumn{1}{c}{ Variável } & $\begin{array}{c}\text { Ciudad } \\
\text { Obregón } \\
\text { Média (DP) }\end{array}$ & $\begin{array}{c}\text { Hermosillo } \\
\text { Média (DP) }\end{array}$ & $\mathbf{t}$ & $\mathbf{p}$ \\
\hline Renda & $785,0(757)$ & $650,0(725)$ & 1,01 & $>0,05\left(^{*}\right)$ \\
Idade & $37,2(15,3)$ & $35,5(15,3)$ & 0,60 & $>0,05\left(^{*}\right)$ \\
Nível educacional & $10,6(4,8)$ & $10,3(4,2)$ & 0,54 & $>0,05\left(^{*}\right)$ \\
Tamanho da família & $4,7(1,3)$ & $4,8(1,4)$ & $-0,52$ & $>0,05\left(^{*}\right)$ \\
\hline
\end{tabular}

(*) diferença estatisticamente não significativa 
nalmente, um modelo de equações estruturais foi especificado e testado, envolvendo a relação entre essas variáveis e o consumo de água.

Nesse modelo foram construídos quatro fatores: motivos de conservação, habilidades de conservação, crenças utilitárias sobre água e consumo de água. Estes fatores resultaram das inter-relações entre cada componente das escalas psicológicas/comportamentais. Exigem-se cargas lambda altas e significativas $(\mathrm{p}<0,05)$ para cada fator, como evidência de validade convergente de construto, ao mesmo tempo em que se espera valores mais baixos de correlações entre fatores, como indicação de validade discriminante de construto (ver Corral-Verdugo \& Figueredo, 1999). E ainda, um coeficiente estrutural significativo de cada fator psicológico relativo à conservação de água indicaria validade concorrente.

De acordo com o modelo especificado, o consumo de água é diretamente afetado por motivos e habilidades de conservação, crenças utilitárias, posse de aparelhos e utensílios domésticos que consomem água e escassez de água. Além disso, os preditores foram especificados como produzindo inter-correlações entre eles. Considerar este modelo como plausível requeria atender critérios de bondade de ajuste (goodness-of-fit). Foram considerados vários indicadores de bondade de ajuste, incluindo o indicador estatístico, qui qua- drado, e os índices práticos: Índice de Ajuste Não Normatizado de Bentler-Bonnet (IANN, ou Bentler-Bonnet Non Normed Index, NNFI); Índice de Ajuste Comparativo (IAC, ou Comparative Fit Index, CFI), assim como o Índice Raiz Quadrada do Quadrado Médio do Erro de Aproximação (RQQMEA, ou Root Mean Square Error of Approximation, RMSEA) (Browne \& Cudeck, 1993; Byrne, 1994). Um qui quadrado baixo e não-significativo ( $p>0,05)$ é considerado como indicativo de bondade de ajuste. No entanto, como um grande número de casos habitualmente resulta em valores significativos deste indicador, os índices práticos são alternativas melhores (Bentler, 1995). Valores mais altos que 0,90 para IANN e IAC são considerados como evidência de bondade de ajuste (Byrne, 1994), enquanto que são desejáveis valores de RQQMEA próximos de zero. Um valor de RQQMEA menor do 0,08 indica um erro razoável de aproximação em termos de bondade de ajuste (Browne \& Cudeck, 1993).

\section{Resultados}

A Tabela 2 mostra que as quatro escalas usadas para este estudo produziram alfas com um valor mais alto que 0,60 . A escala de consumo de água revela que as atividades em que mais se consome água são tomar banho e lavar pratos. Os valores de itens da escala de crenças utilitárias relativas a

Tabela 2

Médias e consistência interna de comportamentos e escalas disposicionais $(N=500)$

\begin{tabular}{|c|c|c|c|c|c|}
\hline ESCALA/VARIÁVEIS & Média & (DP) & Min. & Max. & Alfa \\
\hline CONSUMO DE ÁGUA & & & & & 0,62 \\
\hline Lavar pratos (minutos) & 4,18 & $(5,95)$ & 0 & 46,6 & \\
\hline Tomar banho de chuveiro (minutos) & 8,72 & $(6,44)$ & 0 & 38,3 & \\
\hline Escovar os dentes (minutos) & 2,36 & $(2,15)$ & 0 & 12,0 & \\
\hline Usar a privada (minutos) & 2,62 & $(1,93)$ & 0 & 16,6 & \\
\hline CRENÇAS UTILITÁRIAS SOBRE ÁGUA & & & & & 0,76 \\
\hline Muita água em nosso estado & 2,92 & $(1,11)$ & 1 & 4 & \\
\hline Água é um recurso barato & 2,65 & $(1,14)$ & 1 & 4 & \\
\hline A ciência resolverá problemas da água & 2,30 & $(1,21)$ & 1 & 4 & \\
\hline Falta d'água: mentira de políticos & 2,51 & $(1,15)$ & 1 & 4 & \\
\hline HABILIDADES DE CONSERVAÇÃO & & & & & 0,65 \\
\hline Lavar carro & 2,51 & $(1,43)$ & 0 & 6 & \\
\hline Regar plantas & 1,83 & $(0,88)$ & 0 & 4 & \\
\hline Tomar banho de chuveiro & 1,52 & $(0,66)$ & 0 & 5 & \\
\hline MOTIVOS DE CONSERVAÇÃO & & & & & 0,73 \\
\hline Sem conservação: sem água & 7,00 & $(4,29)$ & 0 & 10 & \\
\hline Cuidados da família & 7,01 & $(4,29)$ & 0 & 10 & \\
\hline Preservação ambiental & 7,70 & $(2,55)$ & 0 & 10 & \\
\hline Há pouca água em casa & 5,00 & $(3,91)$ & 0 & 10 & \\
\hline
\end{tabular}


água são semelhantes (entre 2,3 e 2,9, em uma escala de 1 a 4), como também os da escala de motivos de conservação. Porém, neste caso, a média do item "eu conservo porque há pouca água em casa” $(5,0)$ é mais baixa do que a média do resto dos itens dessa escala (7,00, 7,01 e 7,70; em uma escala de 0 a 10). Os participantes geraram uma média de 2,5 habilidades de economizar ao lavar um carro e apenas 1,5 maneiras de economizar água ao tomar um banho de chuveiro.

A Tabela 3, por sua vez, mostra que a disponibilidade de água em um das cidades estudadas (Cd. Obregón) é quase o dobro da disponibilidade do líquido na outra cidade (Hermosillo). Os participantes em Cd. Obregón também tinham mais aparelhos e utensílios domésticos que consomem água do que os indivíduos em Hermosillo. Além de consumirem significativamente mais água, os participantes de Cd. Obregón exibiram mais crenças utilitárias e habilidades de conservação relativas a água, mas expressaram níveis mais baixos de motivação pró-ambiental do que os de Hermosillo.

A Figura 1 apresenta os resultados do modelo de consumo de água, usando a representação convencional de modelamento de equações estruturais. Os círculos representam os fatores latentes e os retângulos as variáveis observadas. As setas indicam a direção do fluxo causal. Todo fator latente emerge coerentemente de seus indicadores correspondentes. "Motivos de conservação" emergiu de seus indicadores, referindo-se às razões para economizar água; o fator "habilidades de conservação" foi construído a partir dos resultados das três tarefas efetivas exigidas (lavar pratos, regar plantas, tomar banho de chuveiro). "Crenças utilitárias” foi formado por respostas a itens que concebem água como um recurso ilimitado e barato; e o fator "consumo de água” surgiu dos resultados das observações diretas do uso da água. Em todos os casos, foram produzidas cargas fatoriais altas e significativas $(p<0,05)$ conectando cada fator com seus indicadores, o que é evidência da validade convergente de construto. Como os valores de coeficientes estruturais (conectam os fatores entre si) são mais baixos do que os valores das cargas fatoriais, também concluímos que estas medidas exibem validade discriminante de construto (ver Corral-Verdugo \& Figueredo, 1999).

Essa figura também mostra que o consumo de água foi significativa e positivamente influenciado por crenças utilitárias e pela posse de aparelhos e utensílios domésticos que consomem água, ao passo que motivos e habilidades de conservação e escassez de água afetaram negativamente esse consumo. Motivos de conservação foram positivamente afetados por escassez de água e habilidades de conservação e negativamente influenciados pelas crenças utilitárias. As setas encurvadas indicam covariâncias não-direcionais entre variáveis exógenas independentes neste modelo. Escassez de água e crenças utilitárias foram negativamente relacionadas de modo significativo. Habilidades de conservação covariaram positivamente com escassez de água, bem como com aparelhos e utensílios domésticos que consomem água. Indicadores de bondade de ajuste para este modelo são apresentados na legenda da Figura 1. Como um efeito do tamanho

Tabela 3

Comparação de variáveis entre as cidades estudadas.

\begin{tabular}{lcccc}
\hline \multicolumn{1}{c}{ Variável } & $\begin{array}{c}\text { Cd. Obregón } \\
\text { Média (DP) }\end{array}$ & $\begin{array}{c}\text { Hermosillo } \\
\text { Média (DP) }\end{array}$ & t & p \\
\hline A: disponibilidade de água & & & & \\
Horas por dia & $23,58(3,26)$ & $14,08(8,45)$ & 11,60 & $<0,0001$ \\
\hline B: utensílios/equipamentos que consomem água & & & & \\
Caldeiras & $0,89(0,72)$ & $0,62(0,62)$ & 3,93 & $<0,0001$ \\
Chuveiros & $1,25(0,52)$ & $1,33(0,99)$ & $-0,84$ & $>0,05\left(^{*}\right)$ \\
Privadas & $1,40(0,67)$ & $1,30(0,97)$ & 1,10 & $>0,05\left(^{*}\right)$ \\
Torneiras & $7,40(2,68)$ & $3,95(3,28)$ & 10,10 & $<0,0001$ \\
Coolers ${ }^{\text {a }}$ & $0,57(0,54)$ & $0,87(0,77)$ & $-3,93$ & $<0,0001$ \\
Caixas d'água & $1,59(0,54)$ & $1,35(0,77)$ & 3,10 & $<0,0001$ \\
\hline C: variáveis psicológicas & & & & \\
Consumo de água & $7,36(3,26)$ & $3,02(2,08)$ & 16,80 & $<0,0001$ \\
Crenças utilitárias & $3,27(0,64)$ & $2,41(0,84)$ & 9,91 & $<0,0001$ \\
Habilidades de conservação & $2,21(0,84)$ & $1,88(0,77)$ & 3,88 & $<0,0001$ \\
Motivos de conservação & $3,67(1,42)$ & $7,50(2,23)$ & $-17,10$ & $<0,0001$ \\
\hline
\end{tabular}

(a) Aparato de ventilação/refrigeração que utiliza água para redução de temperatura ambiente em regiões áridas.

(*) Diferença estatisticamente não significativa 


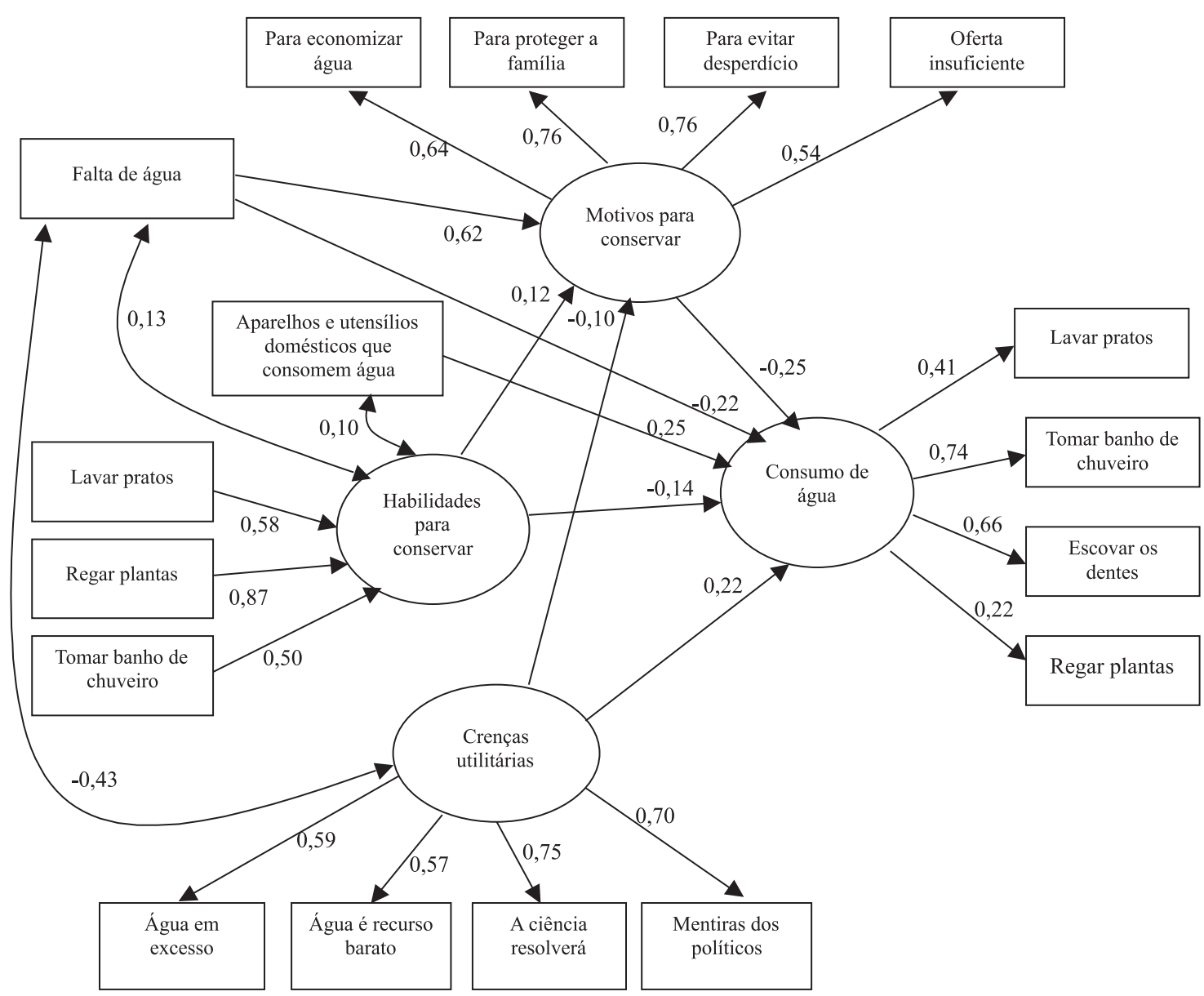

Figura 1. Modelo estrutural de consumo de água predito por fatores disposicionais e situacionais $(\mathrm{N}=500$; método $=$ ML (maximum likelihood, ou verossimilhança máxima); consumo de água: $\mathrm{R}^{2}=0,43$; Bondade de ajuste: $\stackrel{\div}{\div}^{2}=249,7$ (gl = 103), $\mathrm{p}<0,001 ;$ IANN = 0,91; IAC = 0,93; GFI = 1,0; AGFI = 0,91; RQQMEA = 0,04).

da amostra $(\mathrm{N}=500)$, o valor de qui quadrado $(528,4, \mathrm{gl}=263)$ foi significativo $(\mathrm{p}<0,001)$. Entretanto, os valores de IANN e IAC excederam o critério de 0,90 estabelecido para bondade de ajuste. Adicionalmente, RQQMEA produziu um valor $(0,045)$ que também endossa a adequação do modelo testado. O coeficiente de determinação $\left(R^{2}\right)$ deste modelo foi 0,43 , o que significa que os preditores diretos incluídos explicam $43 \%$ da variância do consumo de água.

\section{Discussão}

Como esperado, nosso modelo estrutural demonstrou efeitos significativos diretos e indiretos de variáveis psicológicas e situacionais sobre o consumo de água. Isto significa que tal comportamento depende não somente de fatores contextuais, mas também de variáveis psicológicas, bem como das inter-relações entre estas variáveis (psicológicas e situacionais).
Como mostrado em estudos anteriores (Aitken et al., 1994; Hanke \& De Mare, 1982), nosso modelo revelou que a posse de aparelhos e utensílios domésticos que consomem água promove o seu desperdício. Na medida em que o número desses aparatos aumente nos domicílios, também se elevará o consumo de água. Em nosso estudo, a posse desses aparelhos e utensílios teve uma correlação alta e significativa com a renda familiar $(r=0,52, p<0,0001)$. Uma vez que as pessoas com renda elevada têm os meios financeiros para adquirir e usar esses aparelhos e utensílios, esse resultado revela, em última instância, que a afluência econômica é um instigador do desperdício de água (como relatado por De Oliver, 1999). É interessante notar que esse fator situacional (presença de aparelhos e utensílios que consomem água) também covariou positivamente com habilidades de conservação, o que significa que as pessoas com essas habilidades eram as que tinham mais aparelhos e utensílios que consomem água. Essa covariância, embora significativa, é baixa, e pode estar indi- 
cando que as pessoas de estratos sócio-econômicos mais altos (que possuem aparelhos e utensílios consumidores de água) também têm melhores condições (educação, conhecimento ambiental) para desenvolver um número variado de habilidades, incluindo essas relacionadas com a conservação da água. De qualquer modo, uma vez que é difícil impedir as pessoas (especialmente as mais ricas) de adquirir aparelhos e utensílios que consomem água, a alternativa próambiental é promover a aquisição de dispositivos de conservação ligados a esses aparelhos e utensílios (como recomendado por Geller et al., 1983).

Também de acordo com nosso modelo, a escassez de água teve um efeito direto no seu consumo. Pessoas que vivem sob limitada disponibilidade de água, diminuem o seu uso. Tais limitações também aumentaram, de modo destacado e significativo, a motivação para conservar água: quanto maior a escassez de água, tanto mais o indivíduo desenvolve razões para economizar esse recurso. Uma vez que os motivos de conservação inibem o consumo de água, há também um efeito indireto da escassez de água no seu consumo, através dos motivos de conservação.

Uma outra influência indireta do acesso limitado à água no consumo do líquido, revela-se na relação entre escassez de água e habilidades de conservação. Pessoas que sofrem escassez de água tendem ligeiramente a desenvolver habilidades de conservação. Por sua vez, estas habilidades inibem o consumo de água. Isto revela um outro efeito indireto e inibidor da escassez de água sobre seu consumo, mediado por habilidades de conservação.

Um efeito indireto adicional foi identificado: escassez de água e crenças utilitárias exibem uma covariância saliente e negativa (quanto maior a limitação do acesso à água, tanto menor as crenças utilitárias). Uma vez que estas crenças promovem o consumo de água, há mais um caminho indireto e negativo que conecta escassez de água com consumo de água, neste caso por crenças utilitárias. Assim, nosso modelo parece demonstrar que esse fator situacional não só tem um efeito direto no consumo de água, mas também sobre outras variáveis psicológicas antecedentes. A escassez de água promove o desenvolvimento de tendências de comportamento (motivos, habilidades e crenças) que levam o indivíduo a economizar água.

Que implicações derivam destes resultados? Uma delas é que uma maneira de aumentar tanto a consciência das pessoas sobre problemas de água, como sua tendência para agir de modo responsável em relação ao recurso água, é expor os cidadãos a condições de limitação de água. Como os resultados de nosso estudo mostram, indivíduos que vivem em condições de escassez de água aprendem a dar mais valor a este recurso, desenvolvem suas habilidades para economizá-lo, tendem a não manter crenças utilitárias a respeito, e consomem menos água. Pesquisas futuras deveriam testar a plausibilidade desta explicação.

As variáveis psicológicas também exibiram um padrão de inter-relações significativas. Habilidades de conservação afetaram positiva e diretamente motivos de conservação. Este efeito tinha sido teorizado há algum tempo por De Young (1996), que cunhou o termo "competência de motivação”, implicando que quanto mais habilidosa a pessoa é, mais motivação ela desenvolve como conseqüência de suas habilidades. Assim, educar as pessoas como indivíduos pró-ambientalmente habilitados é uma estratégia duplamente frutífera porque habilidades inibem diretamente o consumo de água e, adicionalmente, promovem motivos para sua conservação.

Por sua vez, crenças utilitárias sobre água, além de promover seu consumo, inibem motivos para sua conservação. Quanto mais os indivíduos pensam na água como um recurso ilimitado, tanto menos eles se sentem compelidos a conservála. Assim, uma mudança nas crenças utilitárias é desejada para aumentar os níveis de motivação para economizar água e a ação de sua conservação. A literatura pertinente mostra que uma forma de mudar crenças ambientais é a educação ambiental (Legault \& Pelletier, 2000), neste caso, dirigida a instigar os cidadãos a desenvolver uma visão pró-ecológica da água como um recurso limitado.

Em resumo, nosso modelo mostrou que o consumo de água está sob a influência combinada de fatores situacionais e disposicionais. Variáveis contextuais, como escassez de água e aparelhos e utensílios que consomem água, afetam significativamente o seu consumo, ao mesmo tempo em que variáveis psicológicas, como motivos ambientais, habilidades e crenças, também influenciam tal comportamento. Além disso, os fatores situacionais promovem o desenvolvimento de tendências psicológicas que predispõem o indivíduo a conceitualizar (crenças), valorizar (motivos) e agir efetivamente (habilidades) no consumo de água.

\section{Referências}

Agras, W., Jacob, R., \& Ledebeck, M. (1980). The California drought: A quasiexperimental analysis of social policy. Journal of Applied Behavior Analysis, 13, 561-570.

Aitken, C. K., McMahon, T. A., Wearing, A. J., \& Finlayson, B. (1994). Residential water use: Predicting and reducing consumption. Journal of Applied Social Psychology, 24, 136-158.

Bentler, P. M. (1995). EQS, Structural Equations Program Manual. Encino, Califórnia: Multivariate Software.

Brown, L. R., and Flavin, C. (1999). A new economy for a new century. In L. Starke (Org.), State of the World. A Worldwatch Institute Report on Progress Toward a Sustainable Society. Nova York: W. W. Norton.

Browne, M. W., \& Cudeck, R. (1993). Alternative ways of assessing model fit. In K. A. Bollen \& J. S. Long (Orgs.), Testing structural equation models (pp. 136-162). Thousand Oaks, Califórnia: Sage.

Byrne, B. M. (1994). Structural equations modeling with EQS and EQS/Windows. Londres: Sage.

Corral-Verdugo, V. (2002). A structural model of pro-environmental competency. Environment \& Behavior, 34, 531-549.

Corral-Verdugo, V., \& Figueredo, A. J. (1999). Convergent and divergent validity of three measures of conservation behavior: The multitraitmultimethod approach. Environment \& Behavior, 31, 805-820.

Crossgrove, W. J., \& Rijsberman, F. R. (2000). World water vision: Making everybody's business. Londres: Earthscan.

Daneshvary, N., Daneshvary, R., \& Schwer, R. K. (1998). Solid-waste recycling behavior and support for curbside textile recycling. Environment \& Behavior, 30, 144-161. 
De Oliver, M. (1999). Attitudes and inaction: A case study of the manifest demographics of urban water conservation. Environment \& Behavior, 31, 372-394

Ewing, G. (2001). Altruistic, egoistic and normative effects on curbside recycling. Environment \& Behavior, 33, 733-764.

Fishbein, M., Middlestadt, S. E., \& Hitchcock, P. J. (1991). Using information to change sexually transmitted disease-related behavior: An analysis based on the theory of reasoned action. In J. Wasserheit, S. Aral, K. Holmes \& P. Hitchcock (Orgs.), Research issues in human behavior and sexually transmitted disease in the AIDS era (pp. 243-257). Washington, DC: American Society for Microbiology.

Gardner, G. (2002). The challenge for Johannesburg: Creating a more secure world. In L. Starke (Org.), State of the World 2002. A Worldwatch Institute Report on Progress Toward a Sustainable Society. Nova York: W. W. Norton.

Gatersleben, B., Steg, L., \& Vlek, C. (2002). Measurement and determinants of environmentally significant consumer behavior. Environment \& Behavior, 34, 335-362.

Geller, E. S., Erickson, J. B., \& Buttram, B. A. (1983). Attempts to promote residential water conservation with educational, behavioral and engineering strategies. Population and Environment: Behavioral and Social Issues, 6, 96-112.

Hanke, S. H., \& de Mare, L. (1982). Residential water demand: A pooled time series, cross section study of Malmo, Sweden. Water Resources Bulletin, 18, 621-625.
Instituto Nacional de Geografía e Informática/INEGI (1992). Sistema de consulta para la información censal (SCINCE). México, DF: Autor.

Legault, L., \& Pelletier, L. (2000). Impact of an environmental education program on students' and parents' attitudes, motivation, and behaviours. Canadian Journal of Behavioural Science, 32, 243-250.

López, E., Balboa, H., Igartúa, A., \& Claramunt, R. (1994). Aproximación al estudio de actitudes respecto al ahorro doméstico de agua en Barcelona. In B. Hernández, E. Suárez \& J. Martínez-Torvisco (Orgs.), Interpretación social y gestión del entorno: aproximaciones desde la psicología ambiental (pp. 29-34 ). La Laguna, Tenerife: Universidad de La Laguna.

Middlestadt, S., Grieser, M., Hernández, O., Tubaishat, K., Sanchack, J., Southwell, B., \& Schwartz, R. (2001). Turning minds on and faucets off: Water conservation education in Jordanian schools. Journal of Environmental Education, 32, 37-45.

Oskamp, S. (2000). A sustainable future for humanity? American Psychologist, $55,496-508$.

Prince of Orange and Rijsberman, F. R. (2000). Summary report of the 2nd World Water Forum: from vision to action. Water Policy, 2, 378-395.

Syme, G., Thomas, J., \& Salerian, S. (1983). Can household attitudes predict water consumption? In Institute of Australian Engineers (IAE, Org.), Hidrology and Water Resources Symposium (pp. 53-56). Canberra: Autor.

Somerville, C., \& Briscoe, J. (2000). Genetic engineering and water. Science, 292, 2217.

1 Traduzido de original em língua inglesa por Violeta O. R. Quevedo, psicóloga, assistente social e aluna do Curso de Mestrado em Psicologia da Universidade Federal do Rio Grande do Norte (revisto por José Q. Pinheiro).

Víctor Corral-Verdugo, Ph.D. em Psicologia Ambiental, The University of Arizona, Tucson (EUA), é professor titular na Universidad de Sonora, Hermosillo, México. Endereço para correspondência: Sevilla 6, Residencial Casa Grande, Sección 3, Hermosillo, Sonora, 83240, México. Tel.: (662) 259-2205. Fax: (662) 259-2205. E-mail: corral@rtn.uson.mx 